\title{
HEALTH-RELATED QUALITY OF LIFE ASSESSMENT FOLLOWING RADICAL CYSTECTOMY IN POPULATION OF A SOUTH ASIAN COUNTRY
}

\author{
Nasira Hayat ${ }^{1}$, Suhaib Bin Bilal Hafi ${ }^{1}$, Farhana Badar ${ }^{2}$, Irfan Ahmed ${ }^{3}$ \\ ${ }^{1}$ Department of Internal Medicine, Shaukat Khanum Memorial Cancer Hospital and Research Centre, Lahore, \\ Pakistan, ${ }^{2}$ Department of Cancer Registry, Shaukat Khanum Memorial Cancer Hospital and Research Centre, \\ Lahore, Pakistan, ${ }^{3}$ Horizon Hospital, Lahore, Pakistan \\ Received: 14 August 2018 / Accepted: 25 September 2018
}

\begin{abstract}
Purpose: Radical cystectomy is carried out for muscle invasive bladder (BL) cancer, which greatly affects the quality of life. The objective of our study was to assess the health-related quality of life (HRQoL) in patients following radical cystectomy.

Methods: A retrospective review was done in patients that underwent radical cystectomy between July 2009 and November 2014 at Shaukat Khanum Memorial Cancer Hospital and Research Centre, Lahore, Pakistan. HRQoL assessments of 34 patients done during clinical psychology review were included in this study. HRQoL was assessed using functional assessment of cancer therapy-BL questionnaire.
\end{abstract}

Results: Low scores were observed in physical well-being and emotional well-being domains with mean scores of 7.32 and 7.97, respectively. Patients scored high on social and family well-being, functional well-being and additional concerns, with mean scores of 24.9, 17.24 and 21.73 , respectively.

Conclusions: Psychological evaluation and rehabilitation should be an integral part of surveillance after radical cystectomy.

Key words: Bladder cancer, functional assessment of cancer therapy-bladder, health-related quality of life, radical cystectomy, South Asian

\section{Introduction}

Radical cystectomy is the standard of care for muscle invasive bladder (BL) cancer. After cystectomy, various methods are used for urinary diversion. Commonly ileal conduit, continent cutaneous diversion and orthotropic neo-BL reconstruction are performed. Although cancer control is similar with various modes of diversion, each one of these carries their own risks and benefits.

BL conservative approaches have been used in selected cases with possibly better-preserved quality of life, ${ }^{[1]}$ but there have been no comparative prospective clinical trials regarding oncological outcome. ${ }^{[2]}$

Correspondence: Dr. Suhaib Bin Bilal Hafi, Department of Internal Medicine, Shaukat Khanum Memorial Cancer Hospital and Research Centre, Lahore, Pakistan.

Email: suhaibbilal@skm.org.pk
Health-related quality of life (HRQoL) refers to the physical, psychological and social domains of health that are influenced by a person's experiences, beliefs, expectations and perceptions. ${ }^{[3]}$ HRQoL has become an important consideration following radical cystectomy. In addition to the morbidity of major surgery, urinary diversion has a great impact on the HRQoL. There is no convincing evidence from existing literature that any particular method of urinary diversion offers superior HRQoL outcomes. Rather, there is growing evidence that good HRQoL can be achieved with patient education and consideration of each patient's clinical and psychosocial situation. ${ }^{[4]}$ HRQoL studies have been reported by leading urooncological centres across the globe. ${ }^{[5]}$ Patients with BL cancer who undergo radical cystectomy have significant declines in multiple components of physical and mental HRQoL. ${ }^{[6]}$ 
Limitations to existing literature are the lack of an available BL cancer-specific, validated instrument to measure HRQoL. In addition, HRQoL differs significantly between countries, cultures and races as the sociocultural milieu in which the patient lives can influence their perception of health and illness. Studies have also begun to explore the role of cultural differences in HRQoL. Some BL cancer-specific HRQoL instruments have been validated including BL cancer index, functional assessment of cancer therapy (FACT)-Vanderbilt cystectomy index and FACT-BL. ${ }^{[4]}$

There is a lack of studies in literature observing HRQoL in South Asian population following radical cystectomy. We used FACT-BL for this particular study to observe HRQoL in Pakistani BL cancer population that underwent radical cystectomy in a cancer hospital setting. To the best of our knowledge, this is the first ever HRQoL assessment study performed in South Asian population following radical cystectomy.

\section{Methods}

This was an observational study which used descriptive statistics. The study was based on retrospective review of existing clinical data from patient that underwent radical cystectomy between July 2009 and November 2014 at the Shaukat Khanum Cancer Hospital and Research Centre, Lahore, Pakistan. This is a rare patient group and all 34 patients that underwent radical cystectomy were included in the study. They were assessed by clinical psychologist in their routine follow-up following surgery. We did not do a sample calculation, as this was an observational study design.

HRQoL was measured using FACT-BL questionnaire. FACT-BL questionnaire was translated into Urdu for the local population understanding. There is no validated Urdu version of FACT-BL available. The translated version was presented to the Scientific Review Committee and Institutional Review Board at SKMCH and RC and was approved.

The FACT-BL is a 39-item quality of life assessment questionnaire, where patients score their responses on a scale from 0 to 4 , with 0 being a point of no concern and 4 being of highest concern. The questionnaire comprises physical well-being (max. score 28), social and family wellbeing (max. score 28), emotional well-being (max. score 24) and functional well-being (max. score 28) domains along with area of additional concerns (max. score 48).

Higher scores indicate poorer quality of life. Patients were asked to fill out this questionnaire at their post-operative hospital visit. The demographics and clinical data were recorded using the electronic hospital information system.

The data and HRQoL scores were recorded and analysed using the SPSS software, version 19.

\section{Results}

A total of 34 patients with BL cancer who underwent radical cystectomy and urinary diversion were evaluated. Of these, 31 were male and three were female patients. The mean age of the patients was 56.3 years (range 34-74 years). The mean time of HRQoL assessment from the time of surgery was 5.9 months (range 1-20 months). $73.5 \%(n=25)$ of patients underwent radical cystectomy and ileal conduit (RC and IC), whereas 26.5\% $(n=9)$ of patients underwent radical cystectomy and neo-BL formation ( $\mathrm{RC}$ and $\mathrm{NB}$ ).

QoL assessment scores on the basis of physical, social, emotional and functional well-being and additional concerns were also noted [Table 1].

Additional concerns were mostly related to the alteration in body shape in patients undergone ileal conduit urinary diversion and lower urinary tract symptoms in patients with neo-BL. The best scores were noted in physical domain with a mean score of 7.32 with lack of energy and pain being the main symptoms [Figure 1]. Patients scored rather poor as far as social and family well-being was concerned, family support, acceptance and deterioration of sex life being the main concerns [Figure 2]. Sadness was the main concern in emotional evaluation [Figure 3]. Enjoyment and recreational activities were effected in most people when functional well being was analyzed [Figure 4].

\section{Discussion}

In BL cancer patients, radical cystectomy is known to be one of the most traumatic cancer operations in terms of psychological stress and alteration in lifestyle. ${ }^{[7]}$ Biggest 
Table 1: QoL assessment scores

\begin{tabular}{l|c|c|c|c|c}
\hline & $\begin{array}{c}\text { Physical } \\
\text { well-being }\end{array}$ & $\begin{array}{c}\text { Social/family } \\
\text { well-being }\end{array}$ & $\begin{array}{c}\text { Emotional } \\
\text { well-being }\end{array}$ & $\begin{array}{c}\text { Functional } \\
\text { well-being }\end{array}$ & $\begin{array}{c}\text { Additional } \\
\text { concerns }\end{array}$ \\
\hline Mean \pm SD & $7.32 \pm 4.41$ & $24.91 \pm 33.15$ & $7.97 \pm 4.38$ & $17.24 \pm 6.27$ & $21.73 \pm 5.90$ \\
\hline Range & $0-19$ & $8-21$ & $0-18$ & $0-28$ & $11-35$ \\
\hline
\end{tabular}

SD: Standard deviation

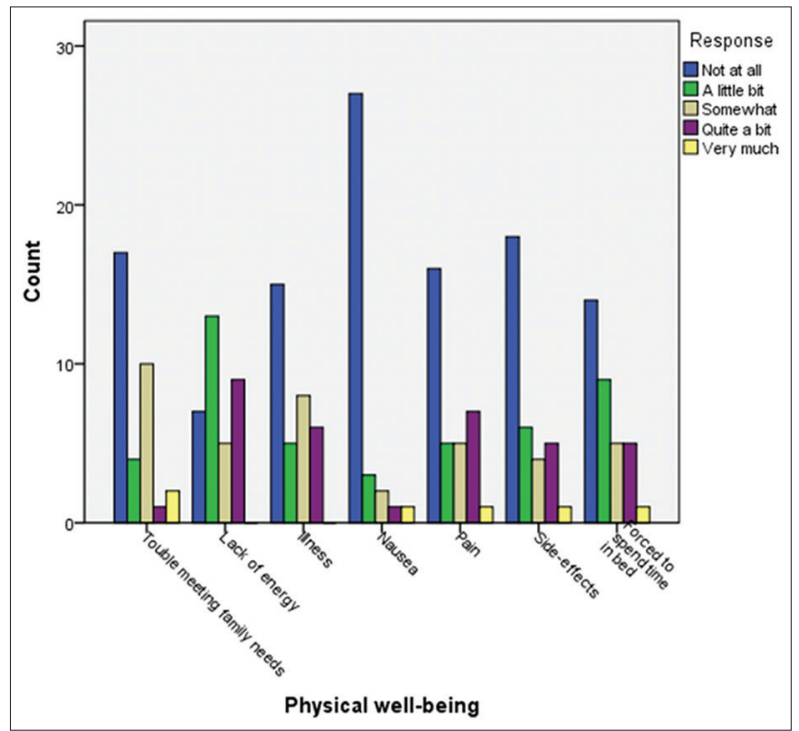

Figure 1: Physical well-being

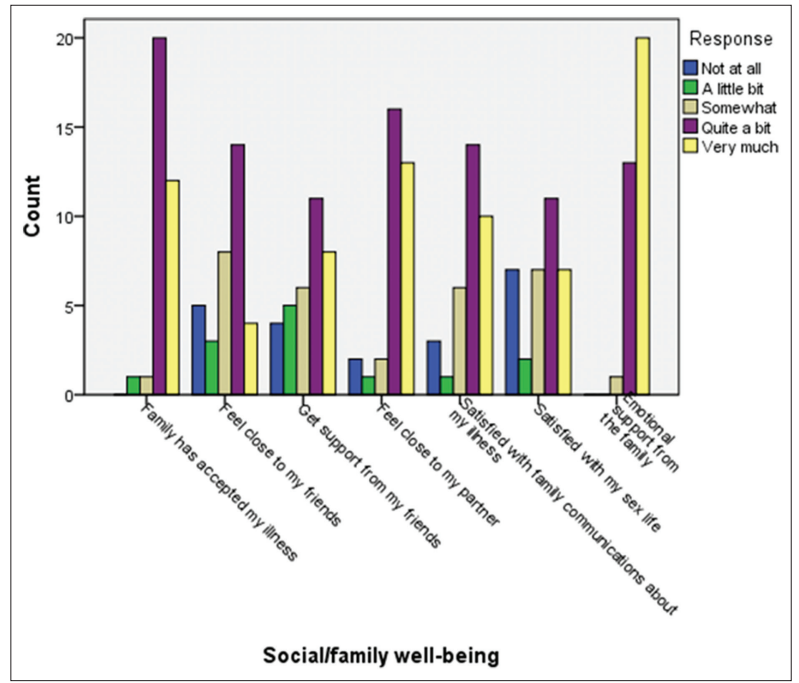

Figure 2: Social and family well-being

determinants of quality of life for an individual are family relationship, general health and finances. Body image and general health certainly play a major role in this.

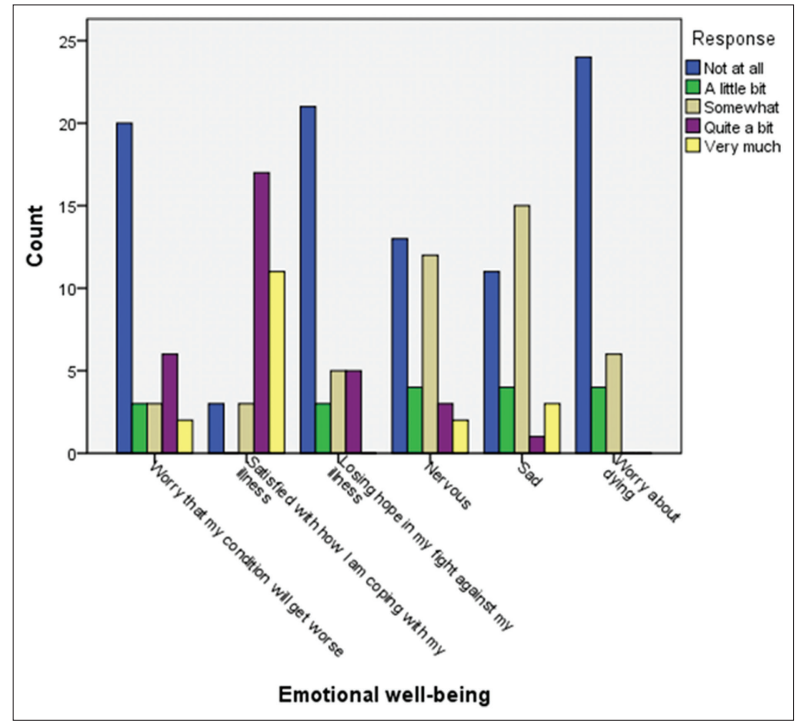

Figure 3: Emotional evaluation

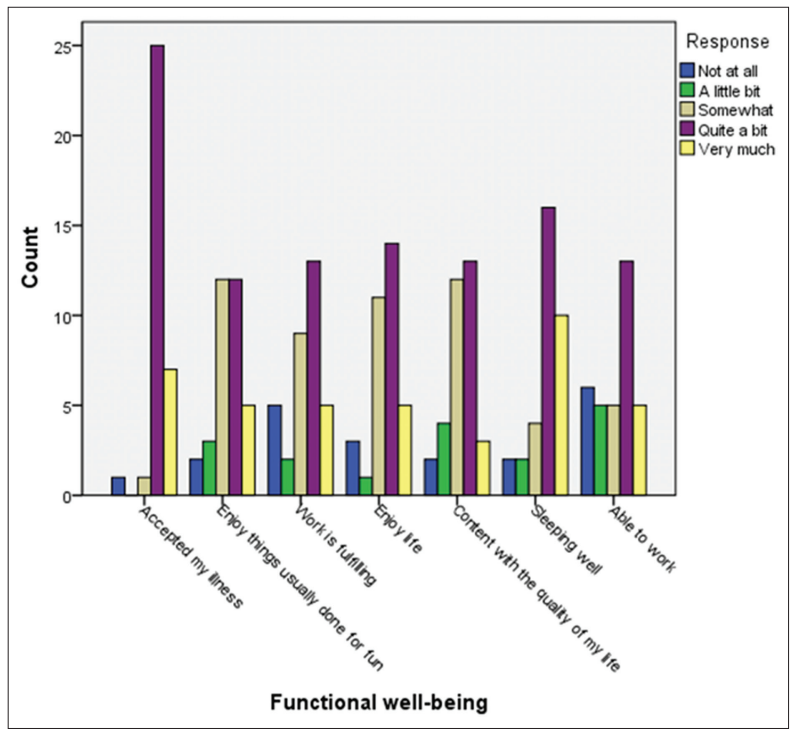

Figure 4: Functional well being

Furthermore, erectile dysfunction, bodily disfigurement, actual or risk of urinary leakage and odour and common 
sequelae of cystectomy and conduit diversion may even lead to marital breakdown. ${ }^{[7]}$ Many factors, for example, an individual's social support system, general and psychological condition of health, disease control and value system play a role in defining the HRQoL of a patient. In another study, FACT-BL domains most negatively affected by surgery were the physical wellbeing and family well-being. ${ }^{[8]}$ These findings in the previous studies are consistent with our results, which demonstrated poor scores in social and family well-being where main concerns were not getting emotional support and acceptance from family. Our patients also reported deterioration in their sex life and concerns secondary to alteration in body image.

Mansson's also reported in their study that fatigue and depression were common among these patients. Many patients reported restlessness, a sense of meaninglessness, a reluctance to ask for help, heightened irritability, gloom and increased sensitivity and nervousness. ${ }^{[7]}$ This was also consistent with our study findings where our patients scored high on feeling sad and nervous. Various studies conducted on HRQoL showed that levels of psychological distress decreased significantly 1 month after surgery compared to pre-operative levels. Palapattu has shown that $45 \%$ of patients demonstrated psychological distress before cystectomy, which decreased to 34\% 1 month after surgery with significant improvements in general distress, depression and anxiety. ${ }^{[9]}$ While recovery may affect QoL and vary from patient to patient, it was demonstrated that psychological and HRQoL stabilise at about 12 months following radical cystectomy. ${ }^{[10]}$ Hence, it is safe to assume that with time distress associated with emotional well-being reduced significantly. Meantime of HRQoL assessment in our study was around 6 months after surgery and the scores were generally low in the emotional well-being domains (except sadness and nervousness) with a mean score of 7.97, which could be because the assessments were carried out several months after the surgery.

HRQoL may also be affected by the type of diversion whether that is incontinent ileal conduit or continent diversion in the form of neo-BL/BL reservoir. In general, majority of the patients get satisfied with their diversion and adapt well socially, physically and psychologically, and the type of urinary diversion does not appear to be associated with differential quality of life. ${ }^{[1]]} \mathrm{Neo}-\mathrm{BL}$ patients were more able to adapt to their new life and had a better QoL with regard to self-confidence, rehabilitation and restoration of leisure, professional, travelling and social activities. ${ }^{[12,13]}$ However, other studies indicated that the HRQoL differences between neo-BL and ileal conduit are non-existent or marginal at best, highlighting that the younger age and improved health in general of neo-BL patients may account for the small differences in HRQoL. ${ }^{[14,15]}$ Furthermore, Kikuchi et al. reported in their prospective study that the type of urinary diversion did not appear to be associated with a different HRQoL by general cancer-related assessment. ${ }^{[16]}$ We had patients who had both ileal conduit and neo-BL surgeries performed; however, we did not specifically look into differences in scores on HRQoL between the two groups given the depth of research done on this already as demonstrated above. Furthermore, a prospective study designed to look into this would have been a more appropriate study method.

A major obstacle in assessing the quality is lack of diseasespecific HRQoL instrument, which could universally compare patients after urinary diversion. Given the limitations of HRQoL research for urinary diversion, a number of research studies fail to demonstrate superiority of any diversion type with regard to psychological or HRQoL. In addition, over a period, better, surgical techniques and various types of urinary diversions have developed to attain better oncological outcome; however, HRQoL remains major concern in such patients.

We do recognize that the study done in one institute may not represent the whole South Asian population, but our study is the first of its kind in this population and further studies are required involving other institutions for the results to be generalised. Limitation of the study was that it was a retrospective review. A prospective study with follow-up reviews of HRQoL would be a better design to focus on different types of surgeries and their impact on specific domains of well-being and their relation with time.

\section{Conclusions}

Psychological evaluation focusing on HRQoL should be done after a major surgical procedure such as radical cystectomy. It is more important when such a procedure affects the body image. Psychological evaluation and rehabilitation should be an integral part of surveillance 
after radical cystectomy. Based on this family and social support should be established for better long-term care of patients with radical cystectomy. Furthermore, there is a need for carrying out prospective research on HRQoL assessment in patients undergoing radical cystectomy in South Asian population to look into the relation of different types of the urinary diversions and their impact of the specific domains of well-being to better address post-operative follow-up care and support.

\section{Conflict of Interest}

The authors declare that they have no conflict of interest.

\section{References}

1. Zietman AL, Sacco D, Skowronski U, et al. Organ conservation in invasive bladder cancer by transurethral resection, chemotherapy and radiation: Results of a urodynamic and quality of life study on long-term survivors. J Urol 2003;170:1772-6.

2. Roca JF. Bladder preservation protocols in the treatment of muscle invasive bladder cancer. Cancer Control 2004; 11:358-63.

3. Testa MA, Simonson DC. Assessment of quality-of-life outcomes. N Engl J Med 1996;334:835-40.

4. Shih C, Porter MP. Health-related quality of life after cystectomy and urinary diversion for bladder cancer. Adv Urol 2011;2011:5.

5. Park J, Ahn H. Radical cystectomy and orthotopic bladder substitution using ileum. Korean J Urol 2011;52:233-40.

6. Winters BR, Wright JL, Holt SK, et al. Health related quality of life following radical cystectomy: Comparative analysis from the medicare health outcomes survey. J Urol 2018;199:669-75.
7. Månsson A, Månsson W. When the bladder is gone: Quality of life following different types of urinary diversion. World J Urol 1999; 17:211-8.

8. Yuh B, Butt Z, Fazili A, et al. Short-term quality-oflife assessed after robot-assisted radical cystectomy: A prospective analysis. BJU Int 2009;103:800-4.

9. Palapattu GS, Haisfield-Wolfe ME, Walker JM, et al. Assessment of perioperative psychological distress in patients undergoing radical cystectomy for bladder cancer. J Urol 2004;172:1814-7.

10. Kulaksizoglu H, Toktas G, Kulaksizoglu IB, et al. When should quality of life be measured after radical cystectomy? Eur Urol 2002;42:350-5.

11. Hart S, Skinner EC, Meyerowitz BE, et al. Quality of life after radical cystectomy for bladder cancer in patients with an ileal conduit, cutaneous or urethral kock pouch. J Urol 1999;162:77-81.

12. El-Sayed M, El-Azab A, El- Gammal M. Quality of life in bladder cancer patients treated with radical 12 . cystectomy and orthotopic bladder reconstruction versus bladder preservation protocol. J Cancer Sci Ther 2013;5:190-3.

13. Hobisch A, Tosun K, Kinzl J, et al. Quality of life after cystectomy and orthotopic neobladder versus ileal conduit urinary diversion. World J Urol 2000;18:338-44.

14. Dutta SC, Chang SC, Coffey CS, et al. Health related quality of life assessment after radical cystectomy: Comparison of ileal conduit with continent orthotopic neobladder. J Urol 2002;168:164-7.

15. Autorino R, Quarto G, Di Lorenzo G, et al. Health related quality of life after radical cystectomy: Comparison of ileal conduit to continent orthotopic neobladder. Eur J Surg Oncol 2009;35:858-64.

16. Kikuchi E, Horiguchi Y, Nakashima J, et al. Assessment of long-term quality of life using the FACT-BL questionnaire in patients with an ileal conduit, continent reservoir, or orthotopic neobladder. Jpn J Clin Oncol 2006;36:712-6. 